\title{
BMJ Open Population-based case-control study of the effect of sun glare on pedestrian fatalities in Taiwan
}

\author{
Hon-Ping Ma, ${ }^{1,2,3}$ Ping-Ling Chen, ${ }^{1}$ Shang-Ku Chen, ${ }^{1,2}$ Liang-Hao Chen, ${ }^{1,4}$ \\ Vaclav Linkov, ${ }^{5}$ Chih-Wei Pai ${ }^{1}$
}

To cite: Ma H-P, Chen P-L, Chen S-K, et al. Populationbased case-control study of the effect of sun glare on pedestrian fatalities in Taiwan. BMJ Open 2019;9:e028350. doi:10.1136/ bmjopen-2018-028350

- Prepublication history and additional material for this paper are available online. To view these files, please visit the journal online (http://dx.doi. org/10.1136/bmjopen-2018028350).

Received 04 December 2018 Revised 25 July 2019 Accepted 31 July 2019

Check for updates

(C) Author(s) (or their employer(s)) 2019. Re-use permitted under CC BY-NC. No commercial re-use. See rights and permissions. Published by BMJ.

${ }^{1}$ Graduate Institute of Injury Prevention and Control, College of Public Health, Taipei Medical University, Taipei, Taiwan ${ }^{2}$ Department of Emergency Medicine, Shuang-Ho Hospital, Taipei Medical University, Taipei, Taiwan

${ }^{3}$ Department of Emergency Medicine, School of Medicine,

Taipei Medical University, Taipei, Taiwan

${ }^{4}$ Department of Emergency Medicine, Wan-Fang Hospital, Taipei Medical University, Taipei, Taiwan

${ }^{5}$ Department of Traffic

Psychology, CDV-Transport

Research Centre, Brno, Czechia

Correspondence to

Dr Chih-Wei Pai;

cpai@tmu.edu.tw

\section{ABSTRACT}

Objectives Sun glare is a serious driving hazard and increases crash risks. Relatively few studies have examined the effects of sun glare on pedestrian fatalities, given that a crash has occurred. The primary objective of this study was to investigate the effect of sun glare on pedestrian fatalities.

Design A population-based case-control study.

Setting Taiwan.

Participants Using the Taiwan National Traffic Crash Data and sunrise and sunset data from the National Oceanic and Atmospheric Administration for the period 2003 to 2016, 100411 pedestrians involved in crashes were identified. Of these crashes, 13355 and 87056 were glare-related (case) and non-glare-related (control) crashes, respectively.

Methods To account for unobserved heterogeneity, mixed logit models were estimated to identify the determinants of pedestrian fatalities.

Main outcome measures Pedestrian fatalities. Results Pedestrians involved in glare-related crashes were more likely to be fatally injured than those in non-glare-related crashes $(\beta=0.527 ; t=3.21)$. Other contributory factors to fatal injuries among pedestrians were older pedestrians ( $\beta=0.553$; $t=2.33)$, male drivers $(\beta=0.324 ; t=2.33)$, older drivers $(\beta=0.218 ; t=2.14)$, intoxicated motorists $(\beta=0.606$; $t=2.85)$, rural roadways $(\beta=0.985$; $t=3.92)$, overtaking manoeuvres $(\beta=0.472$; $t=3.58)$, heavy vehicle crash partners $(\beta=0.248 ; t=2.78)$ and sunset hours $(\beta=0.274 ; t=3.08)$. Walking against traffic appeared beneficial for decreasing injury severity $(\beta=-0.304 ; t=-2.76)$.

Conclusions Sun glare is associated with pedestrian fatalities. Older pedestrians, male drivers, older drivers and intoxicated motorists are prevalent determinants of pedestrian fatalities in glare-related crashes.

\section{INTRODUCTION}

Driving is a highly visual task that involves visual function and processing for establishing effective control over a vehicle. ${ }^{1}$ Research $^{23}$ has suggested that bright sunlight is ideal for driving because it increases the contrast, resolution and luminosity of the surrounding landscape. As a result, however, drivers may misinterpret the speed at which parts of the surrounding landscape are approaching and

\section{Strengths and limitations of this study}

- This is the first nationwide population-based casecontrol study of the associations between pedestrian fatalities and sun glare.

- Glare-related crashes were defined by adjusting vehicle travel direction and orientations of sunrise and sunset.

- Glare-related crashes were defined when the angular distance between the driver's line of sight and the sun was between $0^{\circ}$ and $45^{\circ}$.

- Limitations of this study include data, such as geographical characteristics, unavailable in the two datasets.

underestimate their velocity, prompting them to compensate by accelerating. ${ }^{45}$

Generally, bright sunlight causes temporary blindness when the sun is at a relatively low altitude (ie, just after sunrise or before sunset when the sun is just above the horizon) and its rays fall directly in an individual's line of sight. Using a simulator, Gray and Regan ${ }^{6}$ assessed driving performance in both the absence and presence of a low sun. They reported that sun glare resulted in a significant reduction in the safety margin accepted by drivers, the mean number of crashes was significantly higher during conditions of glare than those without glare, and older drivers exhibited significantly greater reductions in the safety margin than did younger drivers. Another simulation study was conducted by Theeuwes $e t a l,{ }^{7}$ who reported that low glare resulted in their participants (drivers) exhibiting a significant decrease in the ability to detect simulated pedestrians along the roadside. Theeuwes $e t a l^{7}$ also revealed that older participants reduced their driving speed the most and exhibited the largest decrease in successful pedestrian detection.

Churchill et $a l^{8}$ employed a geometric model to examine whether sun glare affects the speeds of drivers on roadways and 
concluded that changes in speed as a result of sun glare affected highway congestion. Jurado-Piña and Pardillo Mayora, ${ }^{9}$ in an investigation of the maximum tolerable sun glare determined that glare occurs when at specific angular distances between a driver's line of sight and the sun; these angular distances are $19^{\circ}$ for a 40-year-old driver and $25^{\circ}$ for a 60 -year-old driver. Anue ${ }^{10}$ suggested that sun glare typically does not occur at angular distances greater than $25^{\circ}$.

Several studies have investigated whether a direct relationship exists between sun glare and crashes by using data from police reports or hospitals. In a longitudinal study of patients who had been hospitalised because of a motor-vehicle crash, Redelmeier and Raza ${ }^{11}$ concluded that bright sunlight was associated with an increased risk of life-threatening crashes in Canada. By analysing police-reported crash data from Arizona, USA, Mitra and Washington ${ }^{12}$ indicated that sun glare was a crucial overlooked variable that could explain intersection crashes and that including this variable improved the explanatory power of statistical models. By linking police-reported crash data from Arizona, USA, with sunrise and sunset data from the National Oceanic Atmospheric Administration (NOAA), $\mathrm{Mitra}^{3}$ concluded that the odds of glare causing a crash were higher on roadways running eastbound and westbound than those running northbound and southbound. Mitra ${ }^{3}$ further indicated that rear-end and angle crashes at signalised intersections were affected by sun glare, but that the severity of motorist injury was unaffected by sun glare.

By analysing the traffic-crash database of Chiba, Japan, Hagita and Mori $^{13}$ revealed that crashes involving pedestrians or bicycles at intersections were more likely when the sun was below $45^{\circ}$ above the horizon in the driving direction. Sun et al, ${ }^{14}$ analysing police-reported crash data in Edmonton, Alberta, Canada, reported similar findings, concluding that sun glare significantly contributed to crash occurrence, especially at intersections. Furthermore, they indicated that the effect of sun glare on crash occurrence during mornings on eastbound roads and evenings on westbound roads is significantly greater during the spring and autumn months and that certain crash types (eg, crashes related to signal violation, failure to yield to pedestrians/cyclists and improper turning and lane changing) are more likely during periods of sun glare. By analysing police-reported crash data, Choi and Singh ${ }^{15}$ revealed that, when compared with other age groups, elderly motorists tend to have a greater propensity for striking other vehicles in conditions of sun glare, especially on roadways that are not physically divided.

According to our literature review, a significant gap remains in the understanding of the relationship between sun glare and pedestrian fatalities, conditional on a pedestrian occurring. The primary research hypothesis of the present study was that pedestrian injury severity increases as visibility decreases (ie, during sun glare). This study therefore investigated whether sun glare is associated with pedestrian fatalities. This study also examined the determinants of pedestrian fatalities in crashes related to sun glare.

\section{MATERIALS AND METHODS \\ Data source}

By using the Taiwan National Traffic Crash Dataset as well as sunrise and sunset data from the NOAA for the period from 2003 to 2016 (National Oceanic Earth System Research Laboratory and Atmospheric Administration, ${ }^{16}$ accessed: 22 August 2018), the current study examined the effect of sun glare on pedestrian fatalities, given that a crash involving a vehicle and pedestrian has occurred. The Taiwan National Traffic Crash Dataset comprises two types of file: crash files and vehicle and victim files. Crash files contain general information on the times and dates of crashes, as well as weather, road conditions, lighting conditions and road types. Vehicle and victim files contain vehicle-related information, such vehicle type, manoeuvres, first point of impact and orientation, as well as driver and casualty information, such as age, sex and injury severity. Injury severity is classified into one of two levels: fatality or injury. Victims who die within 24 hours as a result of a crash are classified as fatalities, whereas victims who sustain injuries, whether mild or severe, are classified as cases of injury.

Daily sunrise and sunset times and orientation are available from the NOAA. The sunrise and sunset orientation data for Taiwan are presented in online supplementary appendix 1. By using the data on the temporal characteristics (ie, time and date) and orientation of vehicle crashes from the Taiwan National Traffic Crash Dataset, pedestrian crashes were matched with the sunrise and sunset data of the NOAA and subsequently classified into glare-related or non-glare-related crashes. The Institutional Review Board affiliated with Taipei Medical University approved our study (IRB\#: N201808071). Personal identification data, such as name or identification number, are not available in the dataset.

A glare-related crash was defined as a crash in which the following two conditions were satisfied: the car was travelling in a direction towards the sunrise or sunset and the angular distance between the driver's line of sight and the sun was between $0^{\circ}$ and $45^{\circ}$. Data on a vehicle's travel orientation (north, south, east or west) are available from the National Traffic Crash Dataset, and data on sun orientation are available from the NOAA. The angular distances were adjusted according to the time of the crash (available from the National Traffic Crash Dataset) and daily sunrise and sunset times (available from the NOAA).

For example, according to the Taiwan National Traffic Crash Dataset, a car-pedestrian crash occurred in Hsinchu City, where a car heading northeast collided with a pedestrian at $06: 18$ on 18 June, 2016. Angular distances from $0^{\circ}$ to $45^{\circ}$ were reported ${ }^{13}$ to cause sun glare and potentially affect traffic safety in Japan. We adopted these same angular distances as the threshold for defining a glare-related crash. According to the NOAA 
website, for this particular (18 June, 2016) and location (Hsinchu City with latitude of $24.778^{\circ} \mathrm{N}$ and longitude of $120.988^{\circ} \mathrm{E}$ ), the sun rose from northeast, and the sunrise and sunset times were at 05:07 and 18:47, respectively. The daytime length for this particular day was 13 hours and $40 \mathrm{~min}$, equivalent to $820 \mathrm{~min}$. The angular distances for sunrise and sunset are $0^{\circ}$ to $180^{\circ}$, respectively; on this particular day, the sun moved $0.2195^{\circ}$ every minute $(180 / 820=0.2195)$. The adopted angular distance of $45^{\circ}$ is equivalent to $205 \mathrm{~min}(45 / 0.2195=205)$; therefore, the glare times transformed from the angular distances of $0^{\circ}$ to $45^{\circ}$ for this particular crash can be between 05:07 and 08:32 (range of $205 \mathrm{~min}$ ). This particular crash was therefore classified as a glare-related crash because the car was headed northeast (which was the direction of the sunrise) and the time of the crash $(06: 18)$ was associated with an angular distance within $0^{\circ}$ to $45^{\circ}$ (ie, times between 05:07 and 08:32).

A flow-chart of the sample selection from the Taiwan Traffic Crash Dataset for the period from 2003 to 2016 is presented in online supplementary appendix 2. A total of 195258 pedestrian casualties from traffic crashes during this period were selected from the dataset. In our sample, each pedestrian was counted. Only crashes involving a single pedestrian were considered in this study; crashes involving two or more pedestrians (accounting for $0.12 \%$ of all pedestrian crashes) were excluded. This study also considered only pedestrian crashes in which the crash partner was a motorcycle, car, taxi or heavy vehicle (eg, bus or coach). Crashes that occurred during adverse weather conditions, such as rain, fog or clouds, were excluded $(n=45712)$. A total of 917 cases had missing data with regard to date, time or vehicle orientation. Because these cases (adverse weather conditions and missing data) were not mutually exclusive, the total number of cases excluded was 45365 , yielding a total of 149839 valid cases of pedestrian casualties. These valid cases were matched with the NOAA sunrise and sunset data.

After crashes that had occurred after sunset or before sunrise ( $n=49428)$ were excluded, 100411 cases of pedestrian casualties remained. Of the 100411 pedestrians that were matched with the sunrise and sunset data from the NOAA, 13355 were glare-related cases (treated as cases), and 87056 were non-glare-related cases (treated as controls).

\section{Definition of variables}

The following demographic data were collected regarding the pedestrians in cases of casualties: sex, age (four groups: $<18,18$ to 40,41 to 64 and $\geq 65$ years), alcohol use (yes: breathalyser test results of $\geq 0.15 \mathrm{mg} / \mathrm{L}$ or blood-alcohol concentration (BAC) level $>0.03 \%$; no: breathalyser test results $<0.15 \mathrm{mg} / \mathrm{L}$ or $\mathrm{BAC}$ level $\leq 0.03 \%$ ), licence status (licensed: with a valid licence; unlicensed: without a valid licence) and pedestrian crossing manner (facing traffic: pedestrians walking towards traffic; back to traffic: pedestrians walking with their backs to traffic; crossing: pedestrians crossing the street).
In Taiwan, those under the age of 18 are identified as teenagers who are unable to legally ride motorcycles or drive cars. Those aged 65 years or older were identified as elderly individuals. The remaining individuals aged 18 to 64 were classified into two even age intervals: 18 to 40 and 41 to 64 years. BAC data were obtained by police who conducted breathalyser tests or who ordered follow-up blood tests at hospitals. According to Taiwanese law, drivers with either breathalyser test $\geq 0.15 \mathrm{mg} / \mathrm{L}$ or BAC level $>0.03 \%$ are considered to be drink-driving. Data from breathalyser tests or BAC levels were available only for motorists and not for pedestrians because, by law, only motorists involved in crashes are required to be tested for alcohol consumption.

The vehicle attribute considered was the crash partner (motorcycle, car, taxi or heavy vehicle, such as a bus or coach). The following road factors were considered: sun glare (yes: affected by sun glare; no: unaffected by sun glare) and crash location (rural: roadways with speed limits of $\geq 51 \mathrm{~km} /$ hour; urban: roadways with speed limits of $\leq 50 \mathrm{~km} /$ hour). Two temporal factors, the month of the crash (spring/summer: March to August; autumn/winter: September to February) and days of the crash (weekday: Monday to Friday; weekend: Saturday or Sunday), were examined.

\section{Patient and public involvement}

The current research analysed national police-reported crash data as well as sunrise and sunset data from the NOAA, which are anonymised datasets. Neither patients nor the public were involved in this study.

\section{Statistical analysis}

The distribution of pedestrian injury severity according to a set of variables (ie, human attributes, environmental factors and vehicle characteristics) is reported in this study. $\chi^{2}$ tests were conducted to examine the association between the independent variables and pedestrian injury severity. Because the dependent variable was binary (fatality vs injury), binary mixed logit models, which allow parameter coefficients to have distributions rather than be fixed across individuals, were estimated. The variables discovered through $\chi^{2}$ testing to be associated with the outcome $(p<0.2)$ were then incorporated into multivariate mixed logit models. To detect multi-collinearity among the variables (all categorical), a $\chi^{2}$ independent test was conducted, and Cramer's $V^{17}$ was estimated. To determine whether sun glare was associated with pedestrian fatalities, one overall model that included sun glare as one of the variables was utilised. One additional model with interaction terms of sun glare with other variables was subsequently estimated.

Mixed logit models were estimated to account for unobserved heterogeneity that may have arisen because of unmeasured variables, such as risk perception, behavioural factors and other socioeconomic factors not available in the police-reported crash data. One example of a behavioural factor is distraction by phone use that 
may result in risk-taking inclinations and consequently an increased risk of injury. ${ }^{18} 19$ Ignoring the effects of unobserved variables may lead to inconsistent estimates in all statistical models. ${ }^{20}$

In the present study, the utility of the injury severity $i$ for a crash $n$ was defined as follows:

$$
\mathrm{IS}_{\mathrm{in}}=\beta_{\mathrm{n}} \mathrm{X}_{\mathrm{in}}+\epsilon_{\mathrm{in}}
$$

where $I S_{i n}$ is an injury-severity function determining the injury-severity category $i$ (fatal or injury) for an individual pedestrian $n ; X_{i n}$ is a vector of the observed variables, such as pedestrian and driver attributes, vehicle characteristics and environmental or temporal variables, $\beta_{n}$ is a vector of the parameters associated with $X_{i n}$; and $\varepsilon_{i n}$ is the error term. The mixed logit model uses $\beta_{n}$ as a vector of estimable parameters, which vary among pedestrians. The variation is observed with density $f(\beta / \theta)$, where $\theta$ is a vector of the parameters of the density distribution. In most applications, mixed models specify that the density $f$ has a continuous distribution, such as a normal, lognormal, triangular or uniform distribution.

Given error terms that are independent and identically Gumbel distributed, ${ }^{21}$ the unconditional probability of one alternative $i$ (from the set of injury-severity categories $I$ ) is the integral of the conditional probability with a multinomial logit form over the parameter $\beta$ of density $f$ :

$$
\mathrm{P}_{\mathrm{in}}=\left(\frac{\exp \left(\beta^{\prime} \mathrm{X}_{\mathrm{in}}\right)}{\sum_{\mathrm{J}=1}^{1} 1+\exp \left(\beta^{\prime} \mathbf{X}_{\mathrm{nj}}\right)}\right) \mathrm{f}(\beta \mid \theta) \mathrm{d} \beta
$$

All parameters are initially assumed to be random; then, their estimated SD are evaluated using a zerobased (asymptotic) t-test for each parameter. One study ${ }^{20}$ pointed out that a simulation-based maximum likelihood with 200 Halton draws may provide a more efficient distribution of draws for numerical integration and requires fewer draws to achieve convergence. We attempted to increase the number of Halton draws, and the results appeared stable with the use of 1000 Halton draws.

\section{RESULTS}

Table 1 lists the distribution of pedestrian injury severity according to a set of variables. Of the 13355 glare-related cases, 329 pedestrians $(2.46 \%)$ sustained fatal injuries, which is higher than those in non-glare-related crashes $(1.83 \%)$. Majority of pedestrian crashes involved motorists with valid licences $(85.65 \%)$, sober motorists $(85.97 \%)$, urban roadways $(89.57 \%)$, pedestrians who were hit while crossing the street $(65.85 \%)$ or non-glare conditions $(86.70 \%)$ or occurred on weekdays $(74.83 \%)$.

Through $\chi^{2}$ testing, the following variables were determined to be associated with the outcome: sun glare; pedestrian and driver sex and age; driver license possession and alcohol consumption; crash month, location and partner; pedestrian movement; car manoeuvre; sunset hours and day of the week. These factors were then incorporated into the mixed logit models.
Table 2 presents the estimation results of the mixed logit model of pedestrian injury severity. We compared the Akaike information criterion (AIC) of the models when determining the optimal distribution and determining whether parameters were fixed or random; male motorists and heavy vehicles as crash partners were determined to be random parameters, with a uniform distribution that appears to provide the best statistical fit.

According to the results listed in table 2, glare-related crashes contributed to fatalities $(t=3.21)$, and the estimated parameter was fixed across all observed pedestrians (the AIC, when fixed, was smaller than when allowed to be random). The parameter implies that glared-related crash was more likely to result in fatal injuries among pedestrians than a non-glare-related crash. Other factors discovered to have fixed effects on observed pedestrians and increase the likelihood of fatal injuries were pedestrians aged 65 years or older, motorists aged 65 years or older, rural roadways, intoxicated motorists, weekend days, overtaking manoeuvres and sunset hours.

The parameter for male motorist appeared to be random, having a normal distribution with a mean of 0.324 and SD of 0.389 (see table 2), indicating that individual pedestrians being struck by male motorists had different parameters. Given the estimates (mean $=0.324$, $\mathrm{SD}=0.389$ ), approximately $79.8 \%$ of pedestrians had a higher probability of sustaining fatal injuries when all other variables remained constant. Another parameter found to have a random effect across the sample of pedestrians was the variable of a heavy vehicle as the crash partner (with a normal distribution). This parameter had a mean of 0.274 and SD of 0.622 (see table 2), indicating that individual pedestrians struck by heavy vehicles have different parameters, with $67.0 \%$ of the distributions resulting in a positive parameter (increasing the likelihood of fatal injuries) and $33.0 \%$ resulting in negative parameter (decreasing the likelihood of fatal injuries).

The direction of travel of pedestrians relative to vehicular traffic was also discovered to affect pedestrian fatalities $(t=-2.76)$. This fixed parameter suggests that pedestrians who faced traffic while walking were less likely to sustain fatal injuries than were those making other movements, such as walking with their backs to traffic or crossing the street.

Table 3 presents the estimation results of the mixed logit model of pedestrian injury severity specifically in glare-related crashes. For this model, the only random parameter (with a uniform distribution) was the variable of a heavy vehicle as the crash partner. The heterogeneous effect indicates that crashes involving heavy vehicles were associated with both higher and lower likelihoods of pedestrians sustaining fatal injuries. We speculate that this heterogeneous effect is caused by variance in the experience level among certain driver groups, with more experienced drivers exhibiting a decreased likelihood of causing fatal injuries. In other 
Table 1 Distribution of pedestrian injury severity according to a set of variables for the period 2003 to 2016

\begin{tabular}{|c|c|c|c|c|}
\hline \multirow{2}{*}{\multicolumn{2}{|c|}{ N }} & \multirow{2}{*}{$\begin{array}{l}\text { Fatality } \\
\text { n (\%) }\end{array}$} & \multirow{2}{*}{$\begin{array}{l}\text { Injury } \\
\mathrm{n}(\%)\end{array}$} & \multirow{2}{*}{$\begin{array}{l}\chi^{2} \text { test } \\
\text { P value }\end{array}$} \\
\hline & & & & \\
\hline Total & 100411 & 1925 (1.92) & 98486 (98.08) & \\
\hline \multicolumn{5}{|l|}{ Sun glare } \\
\hline Yes & 13355 (13.30) & $329(2.46)$ & 13026 (97.54) & $<0.01$ \\
\hline No & 87056 (86.70) & $1596(1.83)$ & 85460 (98.17) & \\
\hline \multicolumn{5}{|l|}{ Pedestrian gender } \\
\hline Male & $50942(50.73)$ & 1015 (1.99) & 49927 (98.01) & 0.08 \\
\hline Female & $49469(49.27)$ & $910(1.84)$ & $48559(98.16)$ & \\
\hline \multicolumn{5}{|l|}{ Driver gender } \\
\hline Male & $53351(53.13)$ & $1132(2.12)$ & 52219 (97.88) & $<0.01$ \\
\hline Female & $47060(46.87)$ & 793 (1.69) & 46267 (98.31) & \\
\hline \multicolumn{5}{|c|}{ Pedestrian age (years) } \\
\hline$<18$ & 3644 (3.63) & $67(1.84)$ & $3577(98.16)$ & $<0.01$ \\
\hline $18-40$ & 25851 (25.75) & $154(0.60)$ & 25697 (99.40) & \\
\hline $41-64$ & 31283 (31.15) & $352(1.13)$ & 30931 (98.87) & \\
\hline$\geq 65$ & 39633 (39.47) & $1352(3.41)$ & 38281 (96.59) & \\
\hline \multicolumn{5}{|l|}{ Driver age (years) } \\
\hline$<18$ & $4458(4.44)$ & $103(2.31)$ & 4355 (97.69) & $<0.01$ \\
\hline $18-40$ & 25808 (25.70) & $295(1.14)$ & 25513 (98.86) & \\
\hline $41-64$ & 32523 (32.39) & $474(1.46)$ & 32049 (98.54) & \\
\hline$\geq 65$ & $37622(37.47)$ & $1033(2.75)$ & 36589 (97.25) & \\
\hline \multicolumn{5}{|l|}{ Driver licence } \\
\hline Licensed & 86007 (85.65) & $1609(1.87)$ & 84398 (98.13) & 0.01 \\
\hline Unlicensed & $14404(14.35)$ & $316(2.19)$ & $14088(97.81)$ & \\
\hline \multicolumn{5}{|c|}{ Alcohol use for driver } \\
\hline No & 86322 (85.97) & $1512(1.75)$ & 84810 (98.25) & $<0.01$ \\
\hline Yes & 14089 (14.03) & $413(2.93)$ & $13676(97.07)$ & \\
\hline \multicolumn{5}{|l|}{ Seasons } \\
\hline Spring/summer & 47671 (47.48) & 914 (1.92) & 46757 (98.08) & 0.99 \\
\hline Autumn/winter & $52740(52.52)$ & $1011(1.92)$ & 51729 (98.08) & \\
\hline \multicolumn{5}{|l|}{ Crash location } \\
\hline Rural & $10475(10.43)$ & $352(3.36)$ & 10123 (96.64) & $<0.01$ \\
\hline Urban & 89936 (89.57) & $1573(1.75)$ & 88363 (98.25) & \\
\hline \multicolumn{5}{|l|}{ Crash partner } \\
\hline Motorcycle & 33221 (33.09) & $643(1.94)$ & 32578 (98.06) & $<0.01$ \\
\hline Car & $45963(45.77)$ & $801(1.74)$ & $45162(98.26)$ & \\
\hline Taxi & 9655 (9.62) & $208(2.15)$ & 9447 (97.85) & \\
\hline Heavy vehicle & $11572(11.52)$ & $356(3.08)$ & 11216 (96.92) & \\
\hline \multicolumn{5}{|c|}{ Pedestrian movement } \\
\hline Facing traffic & 9704 (9.66) & $172(1.77)$ & 9532 (98.23) & $<0.01$ \\
\hline Back to traffic & $24584(24.48)$ & $623(2.53)$ & 23961 (97.47) & \\
\hline Crossing & $66123(65.85)$ & $1130(1.71)$ & 64993 (98.29) & \\
\hline \multicolumn{5}{|l|}{ Car manoeuvre } \\
\hline Straight & 50836 (50.63) & $862(1.70)$ & 49974 (98.30) & $<0.01$ \\
\hline Changing lane & 15625 (15.56) & $257(1.64)$ & 15368 (98.36) & \\
\hline
\end{tabular}


Table 1 Continued

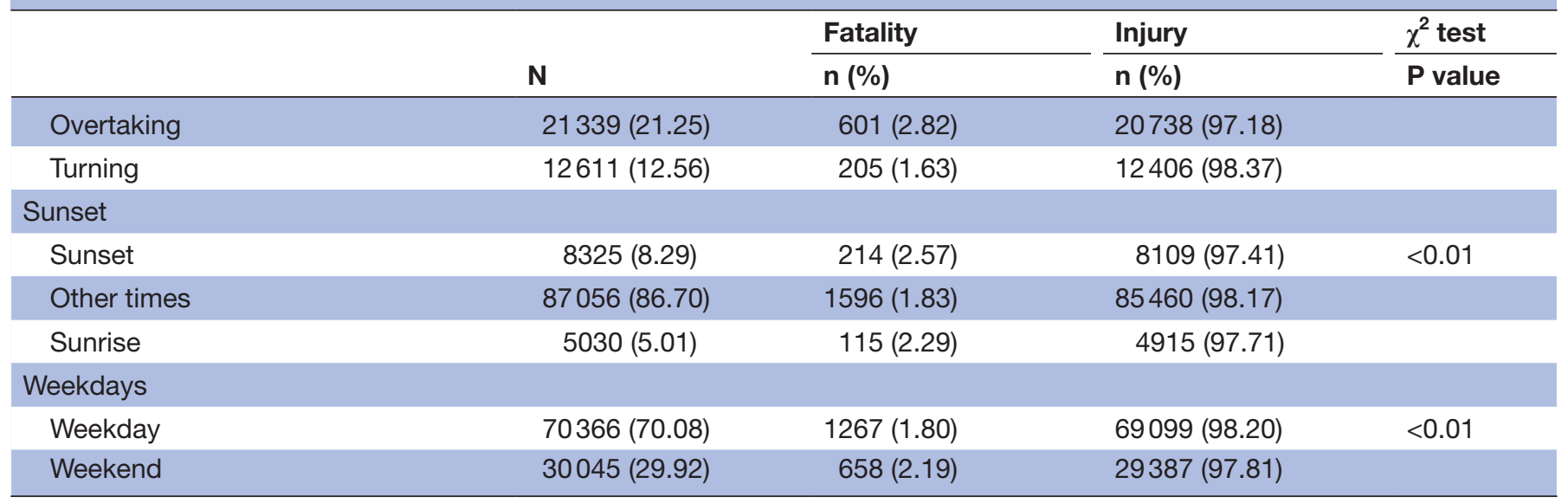

words, heavy vehicles are normally operated by professional drivers, with the majority of drivers in this group being men and middle-aged or older individuals. The heterogeneity of the heavy vehicle partner variable presented supporting estimating a mixed logit model of pedestrian injury severity because such effects are difficult to identify when using a logit framework with numerous interaction terms.

This mixed logit model with interaction terms of glare crashes with other variables highlights several

Table 2 Mixed logit model estimation results for pedestrian injury severity during the period 2003 to $2016^{*}(n=100411)$

\begin{tabular}{lccc}
\hline Variable & Parameter & SE & t value \\
\hline Fatal injury & & & \\
\hline Fixed parameters & & & \\
$\quad$ Constant & -0.531 & 0.215 & -2.47 \\
\hline Glare-related crash & 0.527 & 0.164 & 3.21 \\
\hline Pedestrian facing traffic & -0.304 & 0.110 & -2.76 \\
\hline Pedestrian aged 65+ & 0.553 & 0.237 & 2.33 \\
years & & & \\
\hline Motorist aged 65+ years & 0.218 & 0.102 & 2.14 \\
\hline Rural roadway & 0.985 & 0.251 & 3.92 \\
\hline Intoxicated motorist & 0.606 & 0.213 & 2.85 \\
\hline Weekend & 0.134 & 0.053 & 2.53 \\
\hline Overtaking manoeuvre & 0.472 & 0.132 & 3.58 \\
\hline Sunset & 0.162 & 0.074 & 2.19 \\
\hline Random parameters & & & \\
\hline Male motorist & 0.324 & 0.139 & 2.33 \\
\hline SD of distribution & 0.389 & 0.163 & 2.39 \\
\hline Heavy vehicle partner & 0.274 & 0.110 & 2.49 \\
\hline SD of distribution & 0.622 & 0.290 & 2.14 \\
\hline
\end{tabular}

Restricted log-likelihood (constant only): -8267.1.

Log-likelihood at convergence: -5806.4 .

$\rho^{2}=0.298$.

*The outcome 'injury' is the baseline case with its parameters set at zero. crucial features of glare crashes. For example, several interaction terms were discovered to affect fatalities and fixed across the observed pedestrians: facing traffic $\times$ glare crash, elderly motorist $\times$ glare crash and rural roadway $\times$ glare crash. It appears that facing traffic in a glare-related crash is a protective factor against pedestrian fatalities. Injuries to pedestrians were more likely to be fatal in glare crashes in which the drivers were elderly. Furthermore, in glare crashes that took place in a rural setting, injuries were more likely to be fatal than otherwise.

Table 3 Mixed logit estimation results for pedestrian injury severity with interaction terms of glare-related crashes and other variables* $(n=100411)$

\begin{tabular}{|c|c|c|c|}
\hline Variable & Parameter & SE & $t$ value \\
\hline \multicolumn{4}{|l|}{ Fatal injury } \\
\hline \multicolumn{4}{|l|}{ Fixed parameters } \\
\hline Constant & -0.324 & 0.139 & -2.33 \\
\hline Male motorist & 0.193 & 0.069 & 2.80 \\
\hline Sunset & 0.274 & 0.089 & 3.08 \\
\hline $\begin{array}{l}\text { Pedestrian facing traffic } \times \text { glare } \\
\text { crash }\end{array}$ & -0.439 & 0.126 & -3.48 \\
\hline Pedestrians aged $65+$ years & 0.533 & 0.210 & 2.54 \\
\hline $\begin{array}{l}\text { Motorists aged } 65+\text { years } \times \text { glare } \\
\text { crash }\end{array}$ & 0.432 & 0.143 & 3.02 \\
\hline Rural roadways $\times$ glare crash & 0.684 & 0.190 & 3.60 \\
\hline Intoxicated motorist & 0.461 & 0.154 & 2.99 \\
\hline Weekend & 0.157 & 0.075 & 2.09 \\
\hline Overtaking manoeuvre & 0.329 & 0.121 & 2.72 \\
\hline \multicolumn{4}{|l|}{ Random parameter } \\
\hline Heavy vehicle as crash partner & 0.248 & 0.089 & 2.78 \\
\hline SD of distribution & 0.526 & 0.211 & 2.49 \\
\hline
\end{tabular}

Restricted log-likelihood (constant only): -7302.7.

Log-likelihood at convergence: -5054.6 .

$\rho^{2}=0.308$.

${ }^{*}$ The outcome 'injury' constituted the baseline, with its parameters set at zero. 


\section{DISCUSSIONS}

By using the National Traffic Crash Dataset and sunrise and sunset data from the NOAA, the present study examined whether sun glare was associated with pedestrian fatalities involved in motor vehicle crashes. This research contributes to the growing literature on pedestrian safety as well as fills a major research gap regarding the effect of sun glare on pedestrian fatalities. Regarding methodological contributions, the proposed models offer methodological flexibility to identify individual-specific heterogeneity that may arise as a result of other unmeasured factors related to motorist experience or behavioural or geographical characteristics. When developing intervention strategies for improving pedestrian safety in sun-glare conditions, the heterogeneous effects of certain variables (which cannot be determined with traditional logit models) should be considered.

The empirical contributions of this research are those findings related to the factors affecting pedestrian fatalities. The identification of these risk factors may provide policymakers with information crucial to establishing suitable policies and strategies that may reduce the risk of crashes or severity. The following findings merit further discussion.

Most drivers commute during hours of extreme sun glare. It is therefore not surprising that more crashes occur on roadways that experience a significant portion of traffic during peak morning (sunrise) and afternoon (sunset) hours. The adverse effect of sun glare on crash occurrence has been well-documented in relevant literature. $^{3}{ }^{14}$ However, Mitra ${ }^{3}$ discovered that motorist injury severity was not increased in glare conditions, possibly as a consequence of reduced travel speed. ${ }^{8}$ Such a protective effect of sun glare against associated motorist injury severity does not apply to pedestrians; our study concludes that glare conditions (as indicated in the overall model) are associated with pedestrian fatalities.

In our study, the adverse effect of sun glare was greater on rural roadways where crashes occur at higher speeds and motorists may not expect to encounter pedestrians as often as they would on urban roadways. One commonly-adopted engineering measure is the use of adjust message signs to warn drivers of the risk of sun glare at times and locations (ie, rural roadways) prone to sun glare. $^{9}$

Mitra $^{3}$ noted that the odds of glare causing crashes at intersections were higher during the morning hours and in autumn and winter months in Arizona, USA. In our study, seasons had a negligible effect on pedestrian fatalities. Adding to the research conducted by Hagita and Mori, ${ }^{22}$ who concluded that the rate of pedestrian crashes shortly after sunset was higher than that at any other time in Japan, our study revealed that compared with sunrise, the adverse effect of sun glare on pedestrian fatalities was greater during sunset in Taiwan. Therefore, sun glare during sunset hours may not only increase pedestrian crashes, as indicated by Hagita and Mori, ${ }^{22}$ but also affect the resulting injury severity. Evening commutes are often risky due to fatigue, distraction or other factors unrelated to sun glare. In our study, injuries sustained by pedestrians were more likely to be fatal at sunset than any other daytime of day. Additional research is warranted to examine whether sun glare affects pedestrian fatalities in evening commuting crashes.

Studies have suggested that compared with sober motorists, intoxicated motorists are more likely to leave pedestrians unattended by leaving the crash scene, thereby increasing pedestrian injury severity. Furthermore, alcohol use has been discovered to lead to hit-andruns at night and during the weekend. ${ }^{23}{ }^{24}$ Our study revealed that the combined effects of alcohol consumption and sun glare were associated with pedestrian fatalities. Similar to other studies, ${ }^{23} 24$ our research highlights, in particular, the effect of motorist alcohol consumption on the likelihood of fatality and on the weekends.

Studies have consistently reported that elderly motorists are the group most affected by sun glare. For example, two simulation studies conducted by Gray and $\operatorname{Regan}^{6}$ and Theeuwes $e t a l^{7}$ reported that in conditions of sun glare, older drivers executing a turning manoeuvre demonstrated significantly greater reductions in safety margin than did younger drivers, and older drivers demonstrated the most significant decrease in the ability to successfully detect pedestrians. Studies analysing real-life police-reported crash data ${ }^{15}$ have indicated that in conditions of sun glare, older drivers are more likely to strike other vehicles. Our study contributes to the literature on pedestrian safety by concluding that older motorists, when affected by sun glare, are likely to cause fatal injuries to pedestrians when crashes occur. Advance-warning signs or educational efforts to increase older drivers' awareness of sun-glare conditions may reduce the likelihood of vehicle crashes or pedestrian injury severity.

In our study, no discrepancy was found between injuries in spring/summer and autumn/winter. We speculate that this is primarily because the anticipated effect of sun glare in different seasons may be offset by, for example, typhoons that strike Taiwan in summer and northeast monsoon in winter. Our result differs from those from large country, ${ }^{3}$ where the adverse effect of sun glare was found to be greater in autumn and winter months. Such studies have been conducted in large countries such as the USA, where the climate changes substantially across latitudes, whereas Taiwan is a small island where climate changes little throughout the country.

Considerable work has concluded that older pedestrians are more likely to be fatally or severely injured in crashes, both during daytime and night conditions. ${ }^{25}$ Our research provides ample evidence to suggest that injuries sustained by older pedestrians in crashes caused by sun glare are more likely to be fatal than those sustained by younger pedestrians. Whether the reduced conspicuity of pedestrians (particularly older pedestrians) under conditions of sun glare (twilight) plays a role in this effect is uncertain. However, enhancing the conspicuity of pedestrians with the use of visibility 
aids, not only in night conditions ${ }^{26}$ but also in twilight conditions, may be beneficial for reducing crash risk or severity.

Adding to the research conducted by Sun $e t a l,{ }^{14}$ who reported that crashes related to improper turning or lane changing were more likely to occur during periods of sun glare, the present study reveals an association between the execution of overtaking manoeuvres during periods of sun glare and pedestrian fatalities. Injuries sustained in crashes caused by vehicles executing overtaking manoeuvres are commonly severe because motorists must accelerate to overtake other vehicles. In Taiwan, it is common for motorists to execute overtaking manoeuvres by using roadway shoulders, where pedestrians walk. Motorists should be aware of the risk they pose to pedestrians when overtaking other vehicles, particularly during periods of sun glare.

Studies have reported that facing traffic is beneficial for preventing pedestrian crashes ${ }^{27}$ and reducing the severity of related injuries. ${ }^{25}$ Our study complements these studies by concluding that walking against traffic is associated with decreased injury severity. In these cases, pedestrians' forward views may be well lit and thus be more favourable for pedestrians than walking in other directions. Expressing the necessity of facing traffic while walking along a street, particularly in conditions of sun glare, should be supplemented with information regarding the related safety benefits.

Although it varied among the study pedestrians, being struck by heavy vehicles increased the likelihood of fatal injuries. Although this finding agrees with those of other studies, ${ }^{28}{ }^{29}$ we additionally concluded that the effect of heavy vehicles was greater in conditions of sun glare. Notably, drivers of heavy vehicles tend to be professional. It is unclear whether driving this particular group of vehicles, in combination with undertaking longer hours of travel compared with those undertaken by a car driver, makes such drivers more susceptible to problems related to sun glare than other drivers are. Educational efforts can be directed towards drivers of heavy vehicles regarding driver susceptibility to sun glare, particularly on roadways with pedestrians.

This study had the following research limitations. First, it did not consider the surrounding topography; motorists travelling through areas with buildings or mountains, may be less susceptible to sun glare because the sun is occluded. By analysing geographical and police report data, likely times and locations for sun glare can be predicted, and motorists can be well informed regarding where and when to expect sun glare. Furthermore, we classified crashes as being related to glare when the angular distance between the driver's line of sight and the sun was between $0^{\circ}$ and $45^{\circ}$, a threshold based on research in Japan. ${ }^{13}$ Further research adopting more stringent thresholds of $10^{\circ}, 20^{\circ}$ or $30^{\circ}$ degrees or analysing the changes in this effect for motorists of different ages may attempt to compare their results with ours, as indicated by Jurado-Piña and Pardillo Mayora. ${ }^{9}$
Due to limited funding, we could link only the National Traffic Crash Dataset and NOAA data but not prehospital triage system or hospital data (eg, the National Health Insurance Research Dataset). We recommend that future research link our data to clinical datasets that provide details on injuries (eg, injured body regions, hospitalisation) other than the condition of fatalities examined in this study.

Sun glare is a combined spatiotemporal factor. To broaden our collective understanding of factors of pedestrian safety, collecting spatial and temporal data whenever possible is paramount. The empirical results obtained in this study may be unique to Taiwan because of its unique sunrise and sunset times and orientations. As a result, until additional analyses are conducted using data from other countries to determine whether sun glare is a salient factor of pedestrian fatalities, caution should be exercised in generalising our findings for application in other settings.

Contributors H-PM drafted and revised the manuscript and established the theoretical support for data analyses. P-LC re-analysed the data and interpreted the results. S-KC reviewed relevant literature and analysed the data. L-HC analysed the data. VL edited the manuscript and reviewed relevant literature. C-WP was responsible for study design, contributed to the analysing and interpretation of data, drafted the manuscript and strengthened discussion and conclusion. The final version of the manuscript was read and approved by all contributing authors.

Funding This study was financially supported by grants from the Ministry of Science and Technology, Taiwan (MOST 105-2221-E-038-013-MY3) and Yuan's General Hospital and Taipei Medical University (106YGH-TMU-07). The funders had no role in the design of the study, data collection and analysis, interpretation of data or preparation of the manuscript. Václav Linkov was supported by the Ministry of Education, Youth and Sports within National Sustainability Programme I, a project of Transport R\&D Centre (L01610), on a research grant acquired from the Operational Programme Research and Development for Innovations (CZ.1.05/2.1.00/03.0064).

Competing interests None declared.

Patient consent for publication Not required.

Provenance and peer review Not commissioned; externally peer reviewed.

Data availability statement Data are available in a public, open access repository.

Open access This is an open access article distributed in accordance with the Creative Commons Attribution Non Commercial (CC BY-NC 4.0) license, which permits others to distribute, remix, adapt, build upon this work non-commercially, and license their derivative works on different terms, provided the original work is properly cited, appropriate credit is given, any changes made indicated, and the use is non-commercial. See: http://creativecommons.org/licenses/by-nc/4.0/.

\section{REFERENCES}

1. Owsley C, McGwin G. Vision and driving. Vision Res 2010;50:2348-61.

2. Tversky A, Kahneman D. Judgment under uncertainty: heuristics and biases. Science 1974;185:1124-31.

3. Mitra S. Sun glare and road safety: an empirical investigation of intersection crashes. Saf Sci 2014;70:246-54.

4. Redelmeier DA, Tibshirani RJ. Why cars in the next lane seem to go faster. Nature 1999;401:35-6.

5. Redelmeier DA, Raza S. Optical illusions and life-threatening traffic crashes: a perspective on aerial perspective. Med Hypotheses 2018;114:23-7.

6. Gray R, Regan D. Glare susceptibility test results correlate with temporal safety margin when executing turns across approaching vehicles in simulated low-sun conditions. Ophthalmic Physiol Opt 2007;27:440-50.

7. Theeuwes J, Alferdinck JWAM, Perel M. Relation between glare and driving performance. Hum Factors 2002;44:95-107. 
8. Churchill AM, Tripodis Y, Lovell DJ. Sun glare impacts on freeway congestion: geometric model and empirical analysis. J Transp Eng 2012;138:1196-204.

9. Jurado-Piña R, Mayora JMP, Pardillo Mayora JM. Methodology to predict driver vision impairment situations caused by sun glare. Transp Res Rec 2009;2120:12-17.

10. Anue K. Sun glare detection and visualization. Master's degree in Computer Science. Department of Computer Science. Norwegian University of Science and Technology, 2017.

11. Redelmeier DA, Raza S. Life-threatening motor vehicle crashes in bright sunlight. Medicine 2017;96:e5710.

12. Mitra S, Washington S. On the significance of omitted variables in intersection crash modeling. Accid Anal Prev 2012;49:439-48.

13. Hagita K, Mori K. The effect of sun glare on traffic accidents in Chiba prefecture, Japan. Asian Transport Studies 2014;3:205-19.

14. Sun D, El-Basyouny K, Kwon TJ. Sun glare: network characterization and safety effects. Transp Res Rec 2018;2672:79-92.

15. Choi EH, Santokh S. Statistical assessment of the glare issue human and natural elements. Arlington, VA: Proceeding of 2005 FCSM Conference, Federal Committee on Statistical Methodology, 2005: 1-8.

16. National oceanic earth system research laboratory and atmospheric administration. Available: https://www.esrl.noaa.gov/gmd/grad/ solcalc/ [Accessed 22 Aug 2018].

17. McHugh ML. The chi-square test of independence. Biochemia Medica 2013;23:143-9.

18. Barbieri S, Vettore G, Pietrantonio V, et al. Pedestrian inattention blindness while playing Pokémon go as an emerging health-risk behavior: a case report. J Med Internet Res 2017;19:e86.
19. Chen P-L, Pai C-W. Evaluating the effects of smartphone racing games and other game types on pedestrian's risk-taking inclinations. Comput Human Behav 2018;88:78-83.

20. Bhat CR. Quasi-random maximum simulated likelihood estimation of the mixed multinomial logit model. Transport Res B-Meth 2001;35:677-93.

21. Manski CF, Mcfadden DL. A structural analysis of discrete data with econometric applications. Cambridge, MA: The MIT Press, 1981.

22. Hagita K, Mori K. Traffic accident analysis of sun glare and twilight shortly before and after sunset in Chiba Prefecture, Japan. J East Asia Soc 2015;11:1999-2014.

23. Solnick SJ, Hemenway D. Hit the bottle and run: the role of alcohol in Hit-and-run pedestrian fatalities. J Stud Alcohol 1994:55:679-84.

24. Solnick SJ, Hemenway D. The Hit-and-run in fatal pedestrian accidents: victims, circumstances and drivers. Accid Anal Prev 1995;27:643-9.

25. Chen P-L, Jou R-C, Saleh W, et al. Accidents involving pedestrians with their backs to traffic or facing traffic: an evaluation of crash characteristics and injuries. J Adv Transp 2016;50:736-51.

26. Kwan I, Mapstone J, Cochrane Injuries Group. Interventions for increasing pedestrian and cyclist visibility for the prevention of death and injuries. Cochrane Database Syst Rev 2006;47.

27. Luoma J, Peltola $\mathrm{H}$. Does facing traffic improve pedestrian safety? Accid Anal Prev 2013;50:1207-10.

28. Lefler DE, Gabler HC. The fatality and injury risk of light truck impacts with pedestrians in the United States. Accid Anal Prev 2004;36:295-304.

29. Dong B, Ma X, Chen F. Analyzing the injury severity sustained by non-motorists at mid-blocks considering non-motorists' pre-crash behavior. Transp Res Rec 2018;2672:138-48. 